Editorial

\title{
Sutura y Trasplante Meniscal; lo que todo Traumatólogo Debiera Conocer
}

\author{
David Figueroa ${ }^{1}$ Álvaro Cerda ${ }^{1}$ \\ ${ }^{1}$ Facultad de Medicina Clinica Alemana, Universidad del Desarrollo, Chile \\ Rev Chil Ortop Traumatol 2019;60:33-34.
}

El concepto del menisco como un tejido vestigial y sin importancia, hace algunas décadas planteaba una meniscectomía total abierta como tratamiento ante cualquier lesión meniscal.

Sin embargo, hoy sabemos que sus importantes propiedades y funciones, son determinantes en mantener la biomecánica y la estructura normal de la rodilla, por lo que actualmente el tratamiento de las lesiones meniscales está basado en preservar su integridad.

No obstante, los lineamientos e indicaciones para lograr dicho afán de salvar el menisco, no son del todo claros, por lo que habitualmente nos enfrentamos en ocasiones a situaciones en las cuales nos resulta complejo tomar una decisión terapéutica.

Es por ello que en este editorial trataremos de responder siete preguntas de gran interés y relevancia actual en patología meniscal que todo traumatólogo debería conocer.

1- ¿Cuál es la edad límite para suturar un menisco?

El límite no esta claro, pero bajo los 40 años aumentaría la probabilidad de lograr un buen resultado. En el paciente mayor de esa edad, existiría una disminución de la vascularización meniscal que se volvería mas periférica, ${ }^{1}$ y el menisco tendría características mas degenerativas que disminuirían su potencial de cicatrización.

No obstante, Noyes demostró que pacientes bajo los 20 años, tienen excelentes resultados después de una sutura, situación que también se repitió en su serie de pacientes mayores de 40 años con sutura meniscal, encontrándose ambos grupos asintomáticos a los 51 y 34 meses de seguimiento respectivamente. ${ }^{2,3}$

Una actual revisión sistemática no demostró diferencias significativas en la tasa de fallos en función de rangos etarios (mayor o menor a 20, 25, 30 y 40 años) ${ }^{4}$

Por ello mas importante que la edad cronológica del paciente, sería la evaluación de la integridad y salud del menisco en cada caso, para tomar una decisión definitiva. ${ }^{4,5}$

2- ¿Que tratamiento se plantea en una rotura sintomática radial completa hasta el muro en el cuerpo de menisco externo, en pacientes jóvenes y deportistas?

Address for correspondence David Figueroa, MD, Editor Revista RCHOT, Clínica Alemana de Santiago SA, Vitacura, Región Metropolitana, Chile (e-mail: dhfigueroa@gmail.com).
Las roturas radiales aumentan el estrés de contacto del compartimiento y por ende la progresión a artrosis. ${ }^{6} \mathrm{~A}$ mayor profundidad de la rotura el peak de presión es mayor. ${ }^{7}$.

Esa rotura radial completa es equivalente a una meniscectomía total ya que se pierde la continuidad del anillo meniscal, y por ello deberíamos intentar una sutura.

Una revisión sistemática demostró buenos resultados de la sutura en lesiones radiales a corto plazo (24 meses de seguimiento), faltando aún estudios a largo plazo que reafirmen esa conducta, no obstante hoy en día, la tendencia general es suturar esas lesiones especialmente en pacientes jóvenes y deportistas. ${ }^{8}$

3- ¿ Cuál es el tiempo de evolución límite desde la lesión para considerar suturar una rotura en asa de balde luxada en un paciente joven?

Las lesiones en asa de balde son un desafío ya que se sabe que la extensión de la rotura disminuye la tasa de cicatrización. ${ }^{9}$ Su resolución es quirúrgica pues frecuentemente se luxan causando bloqueo articular.

La literatura reporta buenos resultados en suturas de asas de balde, ${ }^{10}$ aunque hace algunos años se consideraba a las lesiones de más de 6 semanas como crónicas y con peores resultados post reparación que las agudas, ${ }^{11}$ actualmente se han publicado excelentes resultados de suturas en lesiones crónicas (promedio 5 meses de evolución), ${ }^{12}$ e incluso descuidadas por más tiempo (28 meses de evolución $)^{13}$

La decisión no debe basarse únicamente en la cronicidad, sino en la evaluación completa del paciente y la calidad del tejido meniscal. ${ }^{12}$

4. ¿ Que tratamiento se plantea en una rotura degenerativa horizontal en hoja de libro del cuerpo y cuerno anterior-posterior del menisco externo sintomática en un paciente deportista joven?

En el último consenso de ESSKA, 2016 sobre lesiones meniscales degenerativas, se acordó que toda lesión de tipo degenerativa sin antecedente de trauma en pacientes mayores de 35 años, debe someterse a una evaluación clínica e imagenológica, antes de decidir realizar una meniscectomia parcial artroscópica. ${ }^{14}$

Copyright (c) 2019 by Thieme Revinter Publicações Ltda, Rio de Janeiro, Brazil
DOI https://doi.org/ 10.1055/s-0039-1698418. ISSN $0716-4548$.
License terms

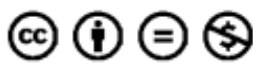


Esos pacientes en particular, por su tipo de practica deportiva y generalmente por sobreuso, pueden acelerar el proceso degenerativo meniscal, que termina en una rotura meniscal que le está impidiendo continuar con su actividad, por lo que la recomendación en la actualidad es la sutura meniscal, con distintas técnicas, siendo considerada la sutura circunferencial como la de mayor consenso. ${ }^{15}$

5. ¿Cuánto tiempo de descarga y que grados de flexión de rodilla es deseable en el postoperatorio de una sutura meniscal sencilla, por ejemplo 2 suturas all-inside en el cuerno posterior de un paciente joven sin otra lesión asociada?

Existen variados esquemas de rehabilitación, el más frecuentemente utilizado es una descarga por 4 a 6 semanas con restricción de la flexión $0-90^{\circ}$ durante el primer mes para luego continuar con flexión progresiva a tolerancia. ${ }^{16}$

El estrés que ejerce la carga axial sobre la reparación va a depender del tipo de rotura y localización, por lo que en roturas verticales se puede permitir carga precoz y en las radiales debemos evitar la carga para proteger la sutura.

Existen además protocolos de rehabilitación acelerada, con menor restricción en cuanto a la carga y flexión; pero que actualmente no han demostrado ser superiores. ${ }^{16}$

6- ¿Cuál es la mejor indicación en una lesión de la raíz posterior del menisco lateral independiente de la edad, peso y actividad física?

Es sabido que una lesión de la raíz es equivalente a una meniscetomía total, ${ }^{17-20}$ por lo que realizar una reinserción con pull out a través de un túnel tibial, puede ser una buena alternativa que podría aliviar los síntomas y restituir así la función meniscal, retrasando la progresión de la artrosis. ${ }^{18}$

Una reciente revisión sistemática de los factores clínicos asociados a malos resultados posterior a una reinserción de raíz meniscal posterior, incluyeron lesiones condrales de alto grado (Outerbridge $\geq 3$ ) preexistentes y varo $\left(>5^{\circ}\right)$. No se encontró asociación con el IMC ni con la edad. ${ }^{19}$

7-¿Cuál es la técnica de fijación de preferencia en un trasplante meniscal?

Existen varias alternativas, no existiendo evidencia hasta la fecha que demuestre superioridad por sobre otra. ${ }^{21}$

Históricamente, la fijación con tacos óseos era lo habitual en menisco interno, y las barras trapezoidales para el menisco externo, ambas técnicas complejas de realizar; pero actualmente la tendencia con demostración tanto biomecánica como de resultados clínicos, es a tunelizar con suturas los cuernos anterior y posterior del menisco a la tibia agregando una capsulodesis para evitar o disminuir la extrusión meniscal. ${ }^{22}$

\section{Bibliografía}

1 Petersen W, Tillmann B. Age-related blood and lymph supply of the knee menisci. A cadaver study. Acta Orthop Scand 1995;66 (04):308-312

2 Noyes FR, Barber-Westin SD. Arthroscopic repair of meniscal tears extending into the avascular zone in patients younger than twenty years of age. Am J Sports Med 2002;30(04):589-600
3 Noyes FR, Barber-Westin SD. Arthroscopic repair of meniscus tears extending into the avascular zone with or without anterior cruciate ligament reconstruction in patients 40 years of age and older. Arthroscopy 2000;16(08):822-829

4 Rothermel SD, Smuin D, Dhawan A. Are Outcomes After Meniscal Repair Age Dependent? A Systematic Review. Arthroscopy 2018; 34(03):979-987

5 Poland S, Everhart JS, Kim W, Axcell K, Magnussen RA, Flanigan DC. Age of 40 Years or Older Does Not Affect Meniscal Repair Failure Risk at 5 Years. Arthroscopy 2019;35(05):1527-1532

6 Badlani JT, Borrero C, Golla S, Harner CD, Irrgang JJ. The effects of meniscus injury on the development of knee osteoarthritis: data from the osteoarthritis initiative. Am J Sports Med 2013;41(06):1238-1244

7 Bedi A, Kelly NH, Baad M, et al. Dynamic contact mechanics of the medial meniscus as a function of radial tear, repair, and partial meniscectomy. J Bone Joint Surg Am 2010;92(06):1398-1408

8 Moulton SG, Bhatia S, Civitarese DM, Frank RM, Dean CS, LaPrade RF. Surgical Techniques and Outcomes of Repairing Meniscal Radial Tears: A Systematic Review. Arthroscopy 2016;32(09):1919-1925

9 Cannon WD Jr, Vittori JM. The incidence of healing in arthroscopic meniscal repairs in anterior cruciate ligament-reconstructed knees versus stable knees. Am J Sports Med 1992;20(02):176-181

10 Ahn JH, Kim KI, Wang JH, Kyung BS, Seo MC, Lee SH. Arthroscopic repair of bucket-handle tears of the lateral meniscus. Knee Surg Sports Traumatol Arthrosc 2015;23(01):205-210

11 Tengrootenhuysen M, Meermans G, Pittoors K, van Riet R, Victor J. Long-term outcome after meniscal repair. Knee Surg Sports Traumatol Arthrosc 2011;19(02):236-241

12 Moatshe G, Cinque ME, Godin JA, Vap AR, Chahla J, LaPrade RF Comparable Outcomes After Bucket-Handle Meniscal Repair and Vertical Meniscal Repair Can Be Achieved at a Minimum 2 Years' Follow-up. Am J Sports Med 2017;45(13):3104-3110

13 Cetinkaya E, Gursu S, Gul M, Aykut US, Ozcafer R. Surgical Repair of Neglected Bucket-Handle Meniscal Tears Displaced into the Intercondylar Notch: Clinical and Radiological Results. J Knee Surg 2018;31(06):514-519

14 Beaufils P, Becker R, Kopf S, et al. Surgical management of degenerative meniscus lesions: the 2016 ESSKA meniscus consensus. Knee Surg Sports Traumatol Arthrosc 2017;25(02):335-346

15 Billières J, Pujol N; and the U45 Committee of ESSKA. Meniscal repair associated with a partial meniscectomy for treating complex horizontal cleavage tears in young patients may lead to excellent long-term outcomes. Knee Surg Sports Traumatol Arthrosc 2019;27(02):343-348

16 O'Donnell K, Freedman KB, Tjoumakaris FP. Rehabilitation Protocols After Isolated Meniscal Repair: A Systematic Review. Am J Sports Med 2017;45(07):1687-1697

17 Allaire R, Muriuki M, Gilbertson L, Harner CD. Biomechanical consequences of a tear of the posterior root of the medial meniscus. Similar to total meniscectomy. J Bone Joint Surg Am 2008;90(09):1922-1931

18 LaPrade RF, Matheny LM, Moulton SG, James EW, Dean CS, Posterior meniscal root repairs: outcomes of an anatomic transtibial pull-out technique. Am J Sports Med 2017;45(04):884-891

19 Jiang EX, Abouljoud MM, Everhart JS, et al. Clinical factors associated with successful meniscal root repairs: A systematic review. Knee 2019;26(02):285-291

20 LaPrade RF, Moulton SG, Cram TR, Geeslin AG, LaPrade CM, Engebretsen L. Meniscal Root Repairs. JBJS Essential Surg Tech 2015;5(04):e19

21 LaPrade RF, Wills NJ, Spiridonov SI, Perkinson S. A prospective outcomes study of meniscal allograft transplantation. Am J Sports Med 2010;38(09):1804-1812

22 Masferrer-Pino A, Monllau JC, Ibáñez M, Erquicia JI, Pelfort X, Gelber PE. Capsulodesis Versus Bone Trough Technique in Lateral Meniscal Allograft Transplantation: Graft Extrusion and Functional Results. Arthroscopy 2018;34(06):1879-1888 